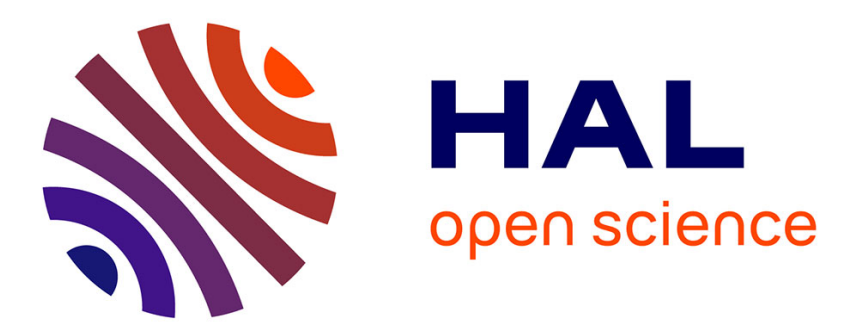

\title{
Conductivity estimates of spherical-particle suspensions based on triplet structure factors
}

Minh Tan Nguyen, Vincent Monchiet, Guy Bonnet, Quy-Dong To

\section{To cite this version:}

Minh Tan Nguyen, Vincent Monchiet, Guy Bonnet, Quy-Dong To. Conductivity estimates of spherical-particle suspensions based on triplet structure factors. Physical Review E : Statistical, Nonlinear, and Soft Matter Physics, 2016, 93, pp.022105. 10.1103/PhysRevE.93.022105 . hal-01283778

\section{HAL Id: hal-01283778 \\ https://hal.science/hal-01283778}

Submitted on 6 Mar 2016

HAL is a multi-disciplinary open access archive for the deposit and dissemination of scientific research documents, whether they are published or not. The documents may come from teaching and research institutions in France or abroad, or from public or private research centers.
L'archive ouverte pluridisciplinaire HAL, est destinée au dépôt et à la diffusion de documents scientifiques de niveau recherche, publiés ou non, émanant des établissements d'enseignement et de recherche français ou étrangers, des laboratoires publics ou privés. 


\title{
Conductivity estimates of spherical-particle suspensions based on triplet structure factors
}

\author{
Minh-Tan Nguyen, Vincent Monchiet, and Guy Bonnet \\ Université Paris-Est, Laboratoire Modelisation et Simulation Multi Echelle, MSME UMR 8208 CNRS, \\ 5 Boulevard Descartes, 77454 Marne-la-Vallée Cedex 2, France \\ Quy-Dong To* \\ Duy Tan University, Institute of Research \& Development, K7/25 Quang Trung, Danang, Vietnam \\ and Université Paris-Est, Laboratoire Modelisation et Simulation Multi Echelle, MSME UMR 8208 CNRS, \\ 5 Boulevard Descartes, 77454 Marne-la-Vallée Cedex 2, France
}

In this paper, we present an estimation of the conductivity of composites constituted of identical spheres embedded in a host material. A family of polarization integral equations for the localization problem is constructed and the operator is then minimized to yield an optimal integral equation. As a result, the corresponding Neumann series converges with the fastest rate and can be used to estimate the effective conductivity. By combining this series and integral approximation, one can derive explicit expressions for the overall property using expansions in Fourier domain. For random hard-sphere systems, relations to structure factors and triplet structure factors have been made and Kirkwood superposition approximation is used to evaluate the effective conductivity, taking into account third-order correlations. This presents an original means to account for the statistical information up to third-order correlation when determining the effective properties of composite materials.

\section{INTRODUCTION}

Determining effective properties of heterogeneous materials from the constituents' has enjoyed a long history $[1,2]$ but is still a very active research area. Due to the variety of microstructures and physical phenomena, different approaches have been proposed to evaluate the overall behavior of the homogenized materials. The usual procedure is to solve the localization problem in a representative volume element (RVE) and to find the relation between the average quantities, such as stress-strain in elasticity or heat flux $(\mathbf{J})$-temperature gradient (E) in conduction, which is the main focus of the present paper. The RVE is chosen so as to represent the material with all heterogeneity details. It can be assimilated to the unit cell for periodic media [3], coated sphere models in micromechanics $[4,5]$, or a sufficiently large material volume for random media [6]. For the latter, bounds on the effective conductivity can be established using variational principles [7] Since the localization problem can be written in the form of integral equations [8], it is possible to expand it into Neumann series and perform a truncation of that series to derive the estimations [9]. This type of approximation is termed "weak contrast expansion" due to the strict convergence condition. Another type of approximation is proposed by Brown [10] based on the polarization and cavity field integral equation. This approach has been shown to estimate effectively the conductivity for some suspensions of spheres [11-14]. However, this series expansion that is derived from the inversion of a conditionally convergent series, can be subject to some subtle issues such as singularities when the two phases are identical and unclear convergence condition that depends on microstructures, especially near the percolation limit [4].

In line with these works, the present paper considers twophase composite materials constituted of identical spherical

*quy-dong.to@u-pem.fr inclusions embedded in a matrix. The arrangement of the spheres can be either periodic, as for cubic distributions, or randomly distributed, as in the case of hard-sphere suspensions. In the periodic settings, a family of integral equations on polarization $p$ is constructed by linearly combining $\boldsymbol{E}$-based and $\boldsymbol{J}$-based polarization equations $[15,16]$. When minimizing the integral operator norm, we obtain an optimal integral equation whose associated Neumann series always converges with fast rate for the whole contrast range. It turns out that the latter presents some similarities with Eyre and Milton's accelerated scheme [17]. Consequently, new estimations of the conductivity based on this quickly convergent series have been derived in this work. Our strategy, which is closely related to the estimation of the remainder of the series [18], is to apply $n$ times the recurrence relation to obtain an integral equation containing $n$ leading terms of the Neumann series. This provides an expression of the effective conductivity tensor involving correlation functions at different orders. Next, approximations can be made to estimate these correlation functions. Compared to previous works in this field, a new original feature is to use information on correlations in the Fourier domain, more particularly the structure factor, and to combine this information with a series expansion whose convergence is optimized. For $n=2$ (two leading terms), numerical results show that such an approach works very well for cubic crystal system at a large range of volume fractions and conductivity contrasts. For random distributions of hard spheres, it is interesting to note that the derived estimation is explicitly related to structure factors at different orders. Our results compare relatively well with the other existing estimations using information on third-order correlations in the literature.

To develop these ideas, the content of our work is divided into four parts. In Sec. II we present the theory of integral equation based on the polarization and its use to estimate the overall conductivity. The treatments of cubic crystal arrangements 
or random materials as well as the approximation of the triplet structure factor are studied. Section III is dedicated to numerical applications of the theory and to comparisons with the available theoretical and numerical results from the literature. Finally, the paper ends with conclusions and remarks in Sec. IV.

\section{HOMOGENIZATION OF THE PERIODIC THERMAL CONDUCTION PROBLEM AND APPROXIMATION METHOD}

A. Class of integral equations based on polarization

In the framework of the homogenization theory, we consider the thermal conduction problem within a cubic unit cell $V=a \times a \times a$, governed by the system of equations [19]

$$
\nabla \times e=0, \quad \nabla \cdot j=0, \quad j=k e,
$$

where the heat flux $j$ and temperature gradient $e$ and conductivity $k$ are $V$-periodic functions of coordinate $x$. The materials are constituted of spherical particles (inclusion $i$ ) embedded in a host material (matrix $m$ ), the conductivity $k$ being constant in each phase and equal to

$$
k=k_{m} \text { in matrix, } \quad k=k_{i} \text { in inclusion. }
$$

The effective conductivity $k_{e}$ is then determined via the linea relation between the cell average, notation $\langle\ldots\rangle_{V}$, of flux $J$ and of temperature gradient $\boldsymbol{E}$ :

$$
J=k_{e} E, \quad J=\langle j(x)\rangle_{V}, \quad E=\langle e(x)\rangle_{V} .
$$

We note here that although the original setting of our problem is the thermal conduction phenomena, the same formulation, Eqs. (1)-(3), and the subsequent results can be applied to diffusion, electrostatic, dielectric, magnetic, or electromagnetic wave in the quasistatic limit [4]. For the latter case, all constants and fields present in the above equations are complex and depend on the excitation frequency $\omega$.

Due to the periodicity of the problem, Eq. (1) can be solved using the Fourier transform technique. We define the two projection operators $\boldsymbol{G}$ and $\boldsymbol{H}$ as the $\Gamma$ 's operator in Ref. [4], p. 247 , by

$$
G(\xi)=\bar{\xi} \otimes \bar{\xi}, \quad H(\xi)=I-\bar{\xi} \otimes \bar{\xi}, \quad \bar{\xi}=\frac{\xi}{\xi} .
$$

Using these complementary projection operators, each vector field $v$ can be decomposed as

$$
v=G v+H v
$$

and it is easy to check that

$$
\begin{aligned}
\nabla \cdot H v & =0, \\
\nabla \times G v & =0 .
\end{aligned}
$$

Therefore, projectors $\boldsymbol{G}, \boldsymbol{H}$ produce irrotational and solenoidal fields. So, we can rewrite the Fourier transform of the first two equations of Eq. (1) in the form

$$
\boldsymbol{H}(\boldsymbol{\xi}) \boldsymbol{e}(\boldsymbol{\xi})=0, \quad \boldsymbol{G}(\boldsymbol{\xi}) \boldsymbol{j}(\boldsymbol{\xi})=0, \quad \forall \xi \neq 0 .
$$

These two equations show that $e(\xi)$ is collinear with the wave vector $\xi$ of norm $\xi$ and $j(\xi)$ is normal to it. In the reciprocal lattice, $\xi$ are the points whose coordinates are given by

$$
\xi_{i}=\frac{2 \pi n_{i}}{a}, \quad n_{i}=0, \pm 1, \pm 2, \ldots, \quad i=1,2,3 .
$$

Now adopting the matrix as the reference material, we define the polarization fields $p$ and $q$,

$$
j=k_{m} e+p, \quad e=j / k_{m}+q,
$$

which are both vanishing in the matrix and finite in the inclusion. Substituting Eq. (8) into Eq. (6) and accounting for Eq. (4) yields the relation

$$
e(\xi)=\boldsymbol{G}(\xi) q(\xi), \quad j(\xi)=\boldsymbol{H}(\xi) p(\xi),
$$

valid for all $\xi \neq 0$. Equivalently, in the physical space we have

$$
e=E+G q, \quad j=J+H p,
$$

where $\boldsymbol{G} \boldsymbol{q}$ and $\boldsymbol{H} \boldsymbol{p}$ represent convolution integrals. In the real space, Eq. (10) can be written as

$$
\begin{aligned}
& e(x)=\boldsymbol{E}+\sum_{\xi \neq 0} \boldsymbol{G}(\xi) q(\xi) e^{-i \xi x}, \\
& j(x)=J+\sum_{\xi \neq 0} \boldsymbol{H}(\xi) p(\xi) e^{-i \xi x} .
\end{aligned}
$$

Making use of the definitions of $p$ and $q$ in relation to $e$ and $j$ and the property $p=-k_{m} q$, we obtain two dual integral equations in terms of $p$

$$
p=\alpha \chi\left(-k_{m} E+G p\right), \quad p=\beta \chi(J+H p) .
$$

In Eq. (12), $\alpha$ and $\beta$ are coefficients depending uniquely on the ratios between material properties,

$$
\alpha=1-\frac{k_{i}}{k_{m}}, \quad \beta=1-\frac{k_{m}}{k_{i}},
$$

and $\chi$ is the characteristic function

$$
\chi=0 \text { in matrix, } \chi=1 \text { in inclusion. }
$$

A class of integral equations based on polarization $p$ can be generated by linearly combining the two equations of Eq. (12). Using two arbitrary coefficients $\lambda_{1}$ and $\lambda_{2}$ with $\lambda_{1}+\lambda_{2}=1$, we obtain the general form

$$
p=\chi A+\chi B p,
$$

in which

$$
\boldsymbol{A}=\left(-\lambda_{1} \alpha k_{m}+\lambda_{2} \beta k_{e}\right) \boldsymbol{E}, \quad \boldsymbol{B}=\lambda_{1} \alpha \boldsymbol{G}+\lambda_{2} \beta \boldsymbol{H} .
$$

As long as the operator norm $\|\boldsymbol{B}\|<1$, we can apply successively the relation Eq. (15) to derive the exact Neumann series expansion of $p$,

$$
\begin{aligned}
p & =\chi \boldsymbol{A}+\chi \boldsymbol{B} p=\chi \boldsymbol{A}+\chi \boldsymbol{B} \chi \boldsymbol{A}+\chi \boldsymbol{B} \chi \boldsymbol{B} \boldsymbol{p}=\ldots \\
& =\chi \sum_{0}^{\infty}(\boldsymbol{B} \chi)^{n} \boldsymbol{A} .
\end{aligned}
$$

This series presents some features that are original. Like in several works using the conductivity tensor of the matrix as reference materials $[5,20]$, this series involves only the characteristic function $\chi$. However, unlike in these previous works, the series can always be chosen to be convergent, as shown thereafter. 
Here, we note that the operator $(\boldsymbol{B} \chi)$ acting on $\phi$ yields

$$
(\boldsymbol{B} \chi) \boldsymbol{\phi}=\sum_{\xi} e^{i \xi \cdot x} \boldsymbol{B}(\xi) \sum_{\xi^{\prime}} \chi\left(\xi-\xi^{\prime}\right) \phi\left(\xi^{\prime}\right),
$$

while the Fourier transform of the constant $A$ admits the simple form

$$
A(\xi \neq 0)=\mathbf{0}, \quad A(\mathbf{0})=A .
$$

Using Eqs. (18) and (19), one can write explicitly all the terms in series Eq. (17). Regarding the convergence rate, the optimal series can be obtained by minimizing $\|\boldsymbol{B}\|$ (hence $\|\boldsymbol{B} \chi\|$ ) in Eq. (16) with $\lambda_{1}$ and $\lambda_{2}$ (see Appendix A). In this case, we have

$$
\begin{aligned}
\left(\lambda_{1}, \lambda_{2}\right) & =\left(\frac{\beta}{\beta-\alpha}, \frac{-\alpha}{\beta-\alpha}\right), \\
\|\boldsymbol{B}\| & =\left|\frac{\alpha \beta}{\beta-\alpha}\right|=\left|\frac{k_{i}-k_{m}}{k_{i}+k_{m}}\right|
\end{aligned}
$$

and

$$
\boldsymbol{A}=\frac{\alpha \beta}{\alpha-\beta}\left(k_{e}-k_{m}\right) \boldsymbol{E}, \quad \boldsymbol{B}=\frac{\alpha \beta}{\alpha-\beta}(\boldsymbol{I}-2 \boldsymbol{G}) .
$$

Certainly, by setting $\left(\lambda_{1}, \lambda_{2}\right)=(1,0)$ and $\left(\lambda_{1}, \lambda_{2}\right)=(0,1)$, we recover the two original equations in Eq. (12). However, the two associated Neumann series, called, respectively, $\boldsymbol{E}$ series (ES) and $J$ series (JS) can diverge if $|\alpha|>1$ or $|\beta|>1$. On the contrary, the optimized series (OS) associated to Eq. (21) having operator $\boldsymbol{B}$ in a form that is similar to the one in Eyre and Milton's accelerated scheme [4,17], always converges with a faster rate. These arguments suggest that estimations based on OS could yield better results than those based on ES or JS [15]

\section{B. Estimation based on Neumann series expansion and integral equation}

To determine the effective property $k_{e}$, we need to find the average polarization $p$ over the inclusion domain $\Omega=f V$. Indeed, from Eq. (8), we have the relation

$$
\langle p\rangle_{\Omega}=f^{-1}\left(k_{e}-k_{m}\right) E \text {. }
$$

Next, from the solution Eqs. (17) and (16), we have

$$
\langle p\rangle_{\Omega}=\left(\lambda_{2} \beta k_{e}-\lambda_{1} \alpha k_{m}\right) \sum_{j=0}^{\infty} \boldsymbol{C}^{j} \boldsymbol{E}
$$

With the effective behavior being isotropic and $f$ being the volume fraction, combining Eqs. (22) and (23) yields

$$
\frac{k_{e}-k_{m}}{\lambda_{2} \beta k_{e}-\lambda_{1} \alpha k_{m}}=\frac{f}{3} \sum_{j=0}^{\infty} \operatorname{tr}\left(C^{j}\right) .
$$

The explicit expressions of $C^{n}$ are given below:

$$
\begin{aligned}
& C^{0}=I, \quad C^{1}=f^{-1} \sum_{\xi} \chi(-\xi) B(\xi) \chi(\xi), \\
& C^{2}=f^{-1} \sum_{\xi} \chi(-\xi) B(\xi) \sum_{\xi^{\prime}} \chi\left(\xi-\xi^{\prime}\right) B\left(\xi^{\prime}\right) \chi\left(\xi^{\prime}\right),
\end{aligned}
$$

$$
\begin{aligned}
\boldsymbol{C}^{j}= & f^{-1} \sum_{\xi^{1}, \xi^{2}, \ldots, \xi^{n}} \chi\left(-\xi^{1}\right) \chi\left(\xi^{1}-\xi^{2}\right) \ldots \chi\left(\xi^{j-1}-\xi^{j}\right) \\
& \times \chi\left(\xi^{1}\right) \boldsymbol{B}\left(\xi^{1}\right) \ldots \boldsymbol{B}\left(\xi^{j}\right) .
\end{aligned}
$$

In numerical practice, we can only keep a finite number $n$ of leading terms of the infinite series, or

$$
\frac{k_{e}-k_{m}}{\lambda_{2} \beta k_{e}-\lambda_{1} \alpha k_{m}}=\frac{f}{3} \sum_{j=0}^{n} \operatorname{tr}\left(C^{j}\right) .
$$

On the other hand, the average $\langle p\rangle_{\Omega}$ can be estimated by making an approximation directly to the integral equation at order $n$,

$$
p=\chi \sum_{j=0}^{n-1}(\boldsymbol{B} \chi)^{j} \boldsymbol{A}+(\chi \boldsymbol{B})^{n} p .
$$

This equation is obtained by repeating the recurrence relation at step $n$ as shown in Eq. (17). By replacing $p$ with its inclusion phase average $\langle p\rangle_{\Omega}$,

$$
p \simeq \chi\langle p\rangle_{\Omega},
$$

in the last integral term of Eq. (27), we obtain an equation for $\langle p\rangle_{\Omega}$,

$$
\langle p\rangle_{\Omega} \simeq\left(\lambda_{2} \beta k_{e}-\lambda_{1} \alpha k_{m}\right) \sum_{j=0}^{n-1} C^{j} E+C^{n}\langle p\rangle_{\Omega} .
$$

Different from the series truncation approximation, the present approximation includes the information of the remainder of the series and is expected to provide better results [18]. Consequently, the final equation for the effective conductivity is the following:

$$
\frac{k_{e}-k_{m}}{\lambda_{2} \beta k_{e}-\lambda_{1} \alpha k_{m}}=\frac{f}{3} \operatorname{tr}\left[\left(I-C^{n}\right)^{-1} \sum_{j=0}^{n-1} C^{j}\right] .
$$

In the numerical applications, we shall use only the integral equation approximation since it provides better results than the series truncation scheme.

\section{Cubic and random distribution of spherical particles}

We now assume that our unit cell $V=a^{3}$ contains $N$ identical nonoverlapping spherical particles of radius $R$ and volume $V_{s}=4 \pi R^{3} / 3$. The shape functions $\chi(\xi)$ become

$$
\begin{aligned}
& \chi(\xi)=\frac{V_{s}}{V} F(\xi) \rho(\xi), \quad \rho(\xi)=\sum_{i=1}^{N} e^{-i \xi \cdot x_{i}}, \\
& F(\xi)=3 \frac{\sin \eta-\eta \cos \eta}{\eta^{3}}, \quad \eta=\xi R,
\end{aligned}
$$

where $\boldsymbol{x}_{i}$ is the location of the sphere numbered $i$ in the cell. In statistical mechanics, the term $F(\xi)$ corresponds to the form factor of the spherical particle and $\rho$ is the microscopic density,

$$
\rho(x)=\sum_{i=1}^{N} \delta\left(x-x_{i}\right) .
$$

For cubic arrangements of spheres, the explicit formulas of $\chi(\xi)$ are known analytically: 
simple cubic (SC),

$$
\chi(\xi)=3 f \frac{\sin \eta-\eta \cos \eta}{\eta^{3}} ;
$$

body-centered cubic (BCC),

$$
\chi(\xi)=\frac{3 f}{2} \frac{\sin \eta-\eta \cos \eta}{\eta^{3}}\left[1+(-1)^{n_{1}+n_{2}+n_{3}}\right] ;
$$

face-centered cubic (FCC),

$$
\begin{aligned}
\chi(\xi)= & \frac{3 f}{4} \frac{\sin \eta-\eta \cos \eta}{\eta^{3}} \\
& \times\left[(-1)^{n_{1}}+(-1)^{n_{2}}+(-1)^{n_{3}}+(-1)^{n_{1}+n_{2}+n_{3}}\right] .
\end{aligned}
$$

These expressions allow us to compute numerically $C^{n}$ at any order $n$. It is also possible to derive analytical expressions for the first two orders $C^{0}=I$ and $C^{1}$, that is a lattice sum. For $C^{1}$, it can be evaluated by keeping some leading fluctuating terms near the origin and approximate the remainder with a continuous integral [15]. Finally, we obtain a closed-form expression that is accurate for finite fraction $f$ and exact at the infinite volume limit $f=0$. However, for higher orders $n>1$, only a numerical computation is available.

For a random distribution (RD), at infinite volume limit (both $N$ and $V \rightarrow \infty$ ) and under ergodicity hypothesis, the tensors $\boldsymbol{C}^{j}$ are identical to their ensemble average, notation $\langle\ldots\rangle$. As a result, we obtain a statistical relations,

$$
\begin{aligned}
\boldsymbol{C}^{1} & =\frac{V_{s}}{V} \sum_{\xi} F(\xi) F(-\xi) \boldsymbol{B}(\xi) S^{(2)}(\xi), \\
\boldsymbol{C}^{2} & =\frac{V_{s}^{2}}{V^{2}} \sum_{\xi, \xi^{\prime}} F(\xi) F\left(\xi^{\prime}\right) F\left(\xi-\xi^{\prime}\right) \boldsymbol{B}(\xi) \boldsymbol{B}\left(\xi^{\prime}\right) S^{(3)}\left(-\xi, \xi^{\prime}\right),
\end{aligned}
$$$$
\text { etc. }
$$

with $S^{(2)}, S^{(3)}$ being the structure factor and the triplet structure factors given by

$$
\begin{aligned}
S^{(2)}(\xi) & =\frac{1}{N}\langle\rho(\xi) \rho(-\xi)\rangle, \\
S^{(3)}\left(\xi, \xi^{\prime}\right) & =\frac{1}{N}\left\langle\rho(\xi) \rho\left(\xi^{\prime}\right) \rho\left(-\xi-\xi^{\prime}\right)\right\rangle, \quad \text { etc. }
\end{aligned}
$$

In the present work, we are not interested in higher-order structure factors that are complicated and hard to determine in practice. Furthermore, in many numerical applications, using only $S^{(2)}(\xi)$ and $S^{(3)}(\xi)$ have already yielded satisfactory results. Posing $\eta=R \xi$ and $\eta^{\prime}=R \xi^{\prime}$ and considering that for large domains we can rewrite the lattice sums Eq. (36) as integrals in the Fourier space, we obtain

$$
\begin{aligned}
C^{1}= & \frac{1}{6 \pi^{2}} \int F(\xi) F(-\xi) \boldsymbol{B}(\xi) S^{(2)}(\xi) d \eta, \\
\boldsymbol{C}^{2}= & \frac{1}{36 \pi^{4}} \iint F(\xi) F\left(\xi^{\prime}\right) F\left(\xi-\xi^{\prime}\right) \\
& \times \boldsymbol{B}(\xi) \boldsymbol{B}\left(\xi^{\prime}\right) S^{(3)}\left(-\xi, \xi^{\prime}\right) d \eta d \eta^{\prime} .
\end{aligned}
$$

For isotropic distributions of hard spheres, we have the following property:

$$
\frac{1}{6 \pi^{2}} \int F^{2}(\xi) S^{(2)}(\xi) d \eta=(1-f)
$$

regardless the local pair distribution $S^{(2)}$. As a result, we can evaluate directly $C^{1}$,

$$
C^{1}=\frac{1}{3}\left(2 \lambda_{2} \beta+\lambda_{1} \alpha\right)(1-f) \boldsymbol{I} .
$$

With this expression, the first-order approximation Eq. (30) with $(n=1)$ based on ES, JS, or OS all coincide with the Clausius-Mossoti equation. Regarding $S^{(3)}$ or the three particle distribution $g^{(3)}$ associated to $\boldsymbol{C}^{2}$, due to the lack of accurate analytical solution, it is usually approximated from statistical information at lower levels, for example, the radial distribution function $g^{(2)}$ or the structure factor $S^{(2)}$. For hard-sphere systems in equilibrium, both $g^{(2)}$ and $S^{(2)}$ are known from the solution of Ornstein-Zernike equation [21] and PercusYevick closure approximation [22,23]. In literature, there are two popular approximations for terms involving third-order correlations. The first one is the Kirkwood superposition approximation (KSA) $[6,24,25]$,

$$
g^{(3)}\left(\boldsymbol{x}, \boldsymbol{x}^{\prime}\right) \simeq g^{(2)}(\boldsymbol{x}) g^{(2)}\left(\boldsymbol{x}^{\prime}\right) g^{(2)}\left(\boldsymbol{x}^{\prime}-\boldsymbol{x}\right),
$$

and the second one has been used for the triplet structure factor [25],

$$
S^{(3)}\left(\xi, \xi^{\prime}\right) \simeq S^{(2)}(\xi) S^{(2)}\left(\xi^{\prime}\right) S^{(2)}\left(\xi-\xi^{\prime}\right) .
$$

Despite the fact that the second approximation provides a direct approximation of $S^{(3)}\left(\xi, \xi^{\prime}\right)$, it violates the core condition, i.e., that it does not avoid the ovelapping of spheres [25]. This can result in a bad estimation of the overall conductivity. The KSA scheme does not suffer from this limitation but it is formulated in physical space and must be translated to $\xi$ space. From the link between $S^{(3)}$ and $g^{(3)}$ and the KSA scheme, we propose the following alternative approximation, justified in Appendix B:

$$
S^{(3)}\left(\xi, \xi^{\prime}\right) \simeq S^{(2)}(\xi) S^{(2)}\left(\xi^{\prime}\right) .
$$

\section{Integral evaluation of $C^{2}$ for random distribution with $S^{(3)}$ approximation}

Regarding $C^{2}$, it is impossible to derive such a simple analytical formula, independently of $S^{(3)}$. However, using approximation Eq. (43), we can convert the sixfold integral

$$
C^{2}=\frac{1}{36 \pi^{4}} \iint T(\eta) T\left(\eta^{\prime}\right) F\left(\left|\eta-\eta^{\prime}\right|\right) \boldsymbol{B}(\eta) \boldsymbol{B}\left(\eta^{\prime}\right) d \eta d \eta^{\prime},
$$

with $T(\eta)=F(\xi) S^{(2)}(\xi)$, into a twofold integral involving $\eta$ and $\eta^{\prime}$. Indeed, we fix first $\eta, \eta^{\prime}$, and $l=\left|\eta-\eta^{\prime}\right|$ and integrate over a circle $\mathcal{C}_{\varphi}$ of radius $R_{\varphi}=\eta^{\prime} \sin \varphi$ (see Fig. 1). By denoting $m_{1}=\lambda_{1} \alpha$ and $m_{2}=\lambda_{2} \beta$, we have

$$
\begin{aligned}
\int_{\mathcal{C}_{\varphi}} \boldsymbol{B}\left(\eta^{\prime}\right) d s \\
=\pi m_{1} R_{\varphi}\left[2 \cos ^{2} \varphi \boldsymbol{G}(\boldsymbol{\eta})+\sin ^{2} \varphi \boldsymbol{H}(\eta)\right] \\
\quad+\pi m_{2} R_{\varphi}\left[\left(2-\sin ^{2} \varphi\right) \boldsymbol{H}(\boldsymbol{\eta})+2 \sin ^{2} \varphi \boldsymbol{G}(\eta)\right] .
\end{aligned}
$$




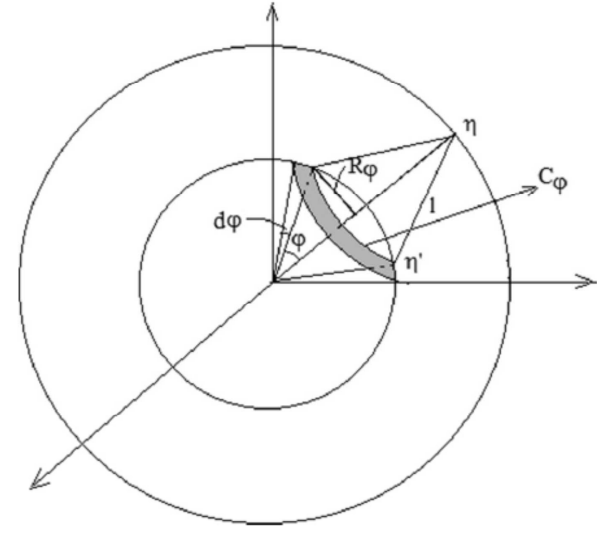

FIG. 1. Integral over a sphere surface.

Now, multiplying $\boldsymbol{B}(\eta)$ with Eq. (45) yields

$$
\begin{aligned}
\boldsymbol{B}(\eta) & \int_{C_{\varphi}} \boldsymbol{B}\left(\eta^{\prime}\right) d s \\
= & 2 m_{1} \pi R_{\varphi}\left[m_{1} \cos ^{2} \varphi+m_{2} \sin ^{2} \varphi\right] \boldsymbol{G}(\boldsymbol{\eta}) \\
& +m_{2} \pi R_{\varphi}\left[m_{2}\left(2-\sin ^{2} \varphi\right)+m_{1} \sin ^{2} \varphi\right] \boldsymbol{H}(\eta) .
\end{aligned}
$$

Next, integrating with $\eta^{\prime}$ running over the sphere surface $S_{\eta^{\prime}}$ of radius $\eta^{\prime}$ and then with $\eta$ running over the sphere surface $S_{\eta}$ of radius $\eta$, we obtain

$$
\begin{aligned}
\int_{S_{\eta^{\prime}}} & \int_{S_{\eta}} F\left(\eta-\eta^{\prime}\right) \boldsymbol{B}(\eta) \boldsymbol{B}\left(\eta^{\prime}\right) d S d S^{\prime} \\
= & 2 \pi m_{1} \eta^{2} \int_{S_{\eta}} \boldsymbol{G}(\eta) d S \int_{0}^{\pi} F(l) \\
& \times\left[m_{2} \sin ^{2} \varphi+m_{1} \cos ^{2} \varphi\right] \sin \varphi d \varphi \\
& +\pi m_{2} \eta^{2} \int_{S_{\eta}} \boldsymbol{H}(\eta) d S \int_{0}^{\pi} F(l) \\
& \times\left[m_{2}\left(2-\sin ^{2} \varphi\right)+m_{1} \sin ^{2} \varphi\right] \sin \varphi d \varphi, \\
l= & \sqrt{\eta^{2}+\eta^{2}-2 \eta \eta^{\prime} \cos \varphi} .
\end{aligned}
$$

With the surface integral identities,

$$
\int_{S_{\eta}} \boldsymbol{G}(\eta) d S=\frac{1}{3} S_{\eta} \boldsymbol{I}, \quad \int_{S_{\eta}} \boldsymbol{H}(\eta) d S=\frac{2}{3} S_{\eta} \boldsymbol{I}, \quad S_{\eta}=4 \pi \eta^{2},
$$

it is now ready to rewrite $C^{2}$ in a simple form,

$$
C^{2}=\frac{2 \boldsymbol{I}}{27 \pi^{2}} \iint T(\eta) T\left(\eta^{\prime}\right) R\left(\eta, \eta^{\prime}\right) \eta^{2} \eta^{\prime 2} d \eta d \eta^{\prime},
$$

with the function $R\left(\eta, \eta^{\prime}\right)$ being defined by

$$
\begin{aligned}
R\left(\eta, \eta^{\prime}\right)= & \int_{-1}^{1} F\left(\sqrt{\eta^{2}+\eta^{\prime 2}-2 \eta \eta^{\prime} t}\right) \\
& \times\left[m_{2}^{2}\left(1+t^{2}\right)+2 m_{1} m_{2}\left(1-t^{2}\right)+m_{1}^{2} t^{2}\right] d t
\end{aligned}
$$

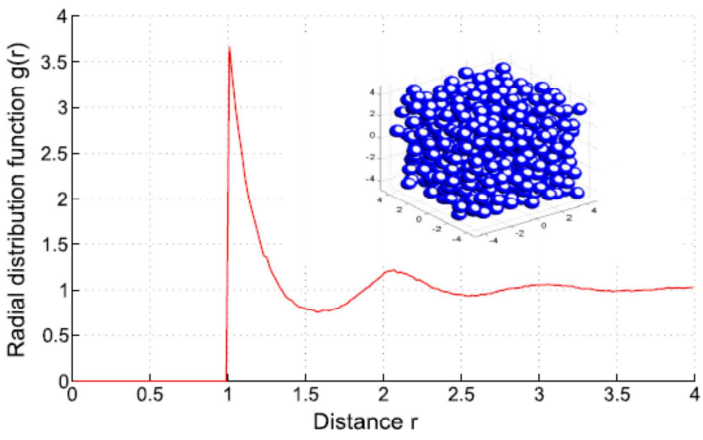

FIG. 2. Radial distribution function of hard sphere in equilibrium at $f=0.4$. The inset is a snapshot of a typical sample.

Numerical examples will show thereafter that the second-order approximation Eq. (30) with ( $n=2)$ and Eq. (44) based on OS provides improved result with respect to the CM estimates.

\section{E. Sample generation and direct computation of $C^{2}$ for random distributions}

As shown above, the calculation of the effective properties based on the integral form encounters difficulties due to the lack of accurate information on $S^{(3)}$. Although many approximations of $S^{(3)}$ (or its dual functions $g^{(3)}, h^{(3)}$ ) have been proposed in the literature [26,27], each approximation scheme is subject to its own limitation and can only capture certain aspects of $S^{(3)}$. In this work, it has been chosen to determine directly $C^{2}$ from the ensemble average of real samples. To this aim, we use the ensemble average version of Eq. (25),

$$
\boldsymbol{C}^{2}=f^{-1}\left\langle\sum_{\xi} \chi(-\xi) \boldsymbol{B}(\xi) \sum_{\xi^{\prime}} \chi\left(\xi-\xi^{\prime}\right) \boldsymbol{B}\left(\xi^{\prime}\right) \chi\left(\xi^{\prime}\right)\right\rangle,
$$

with $\chi(\xi)$ being defined by Eq. (31). All calculations are done in Fourier space including the discrete convolution. The eventdriven molecular dynamics (EDMD) method [28] is used to generate systems of 500 hard spheres in equilibrium at the unit temperature. The final results are then obtain by averaging over 50 samples and will be compared with other estimations. As an example of intermediate result, the radial distribution function (RDF) of the system with a snapshot of a typical sample is shown in Fig. 2.

\section{NUMERICAL APPLICATIONS}

\section{A. Cubic lattice arrangements}

We apply first the approximation schemes to cubic lattice arrangements of spheres: simple cubic (SC) and face-centered cubic (FCC). The reference results for comparison are the exact numerical solutions obtained from a standard FFT numerical method. The details of the latter has been described elsewhere $[18,29]$ and shall not be discussed further here.

From results for SC arrangement in Table I, we find that the OS approximation performs significantly better at all order $n=1$ or 2 and contrast ratio $k_{i} / k_{m}$ when compared with 
TABLE I. Comparison the effective conductivity $\left(k_{e} / k_{m}\right)$ of SC lattice structure between the approximation schemes based on series OS, JS, and ES and the FFT solution.

\begin{tabular}{ccccccc}
\hline \hline$k_{i} / k_{m}$ & $f$ & FFT & JS-1 & OS-1 & JS-2 & OS-2 \\
\hline \multirow{4}{*}{1000} & 0.10 & 1.3416 & 1.3298 & 1.3316 & 1.3306 & 1.3325 \\
& 0.20 & 1.7730 & 1.7422 & 1.7460 & 1.7454 & 1.7514 \\
& 0.30 & 2.3674 & 2.2717 & 2.2781 & 2.2874 & 2.3119 \\
& 0.40 & 3.3389 & 2.976 & 2.986 & 3.0466 & 3.1526 \\
& 0.50 & 6.6290 & 3.958 & 3.976 & 4.247 & 4.718 \\
& 0.10 & 1.2444 & 1.2419 & 1.2429 & 1.2423 & 1.2433 \\
& 0.20 & 1.5337 & 1.5269 & 1.5288 & 1.5284 & 1.5311 \\
10 & 0.30 & 1.8913 & 1.867 & 1.8700 & 1.8738 & 1.8832 \\
& 0.40 & 2.3708 & 2.2798 & 2.2843 & 2.3069 & 2.3408 \\
& 0.50 & 3.1575 & 2.791 & 2.797 & 2.8850 & 3.0009 \\
$k_{i} / k_{m}$ & $f$ & FFT & ES-1 & OS-1 & ES-2 & OS-2 \\
\hline \multirow{6}{*}{0.1} & 0.10 & 0.8765 & 0.8769 & 0.8766 & 0.8768 & 0.8764 \\
& 0.20 & 0.7624 & 0.7634 & 0.7630 & 0.7631 & 0.7623 \\
& 0.30 & 0.6560 & 0.6585 & 0.6581 & 0.6574 & 0.6551 \\
& 0.40 & 0.5541 & 0.5613 & 0.5608 & 0.5578 & 0.5518 \\
& 0.50 & 0.4537 & 0.4710 & 0.4704 & 0.4622 & 0.4487 \\
& 0.10 & 0.8568 & 0.8576 & 0.8572 & 0.8575 & 0.8569 \\
& 0.20 & 0.7263 & 0.7280 & 0.7275 & 0.7276 & 0.7262 \\
0.001 & 0.30 & 0.6052 & 0.6096 & 0.6090 & 0.6080 & 0.6040 \\
& 0.40 & 0.4897 & 0.5011 & 0.5004 & 0.4961 & 0.4855 \\
& 0.50 & 0.3723 & 0.4012 & 0.4005 & 0.3889 & 0.3648 \\
\hline \hline & & & & & &
\end{tabular}

ES and JS approximation. The second-order approximation $(n=2)$ yields better results than the first order $(n=1)$ except for ES series for $k_{i} / k_{m}=10,1000$ and JS series for $k_{i} / k_{m}=$ $0.1,0.001$ (not shown in Table I). This can be explained by the fact that the associated series diverge, making higher-order approximation meaningless. For $k_{i} / k_{m}=0.1,0.001$, there is little difference between the approximation schemes and the exact results. At very high contrast ratio $k_{i} / k_{m}=1000$ and very high volume fraction, the series approximations work less well. The best one, OS approximation, is only acceptable at $f \leqslant 0.4$. It means that a higher-order approximation $n>2$ is needed to achieve a better precision, for example, within $5 \%$ error.

Figure 3 for FCC arrangement shows that the OS approximation still performs very well for $n$ as low as $2, k_{i} / k_{m}$ as high as 100 and volume fraction up to $f=0.6$. While the second-order approximation has significantly improved the first order, there is a very slight difference between the third order and the second order. This agrees with the very fast convergence rate of the OS series; i.e., the final solution is not far from the sum of several initial terms in the series.

All the results obtained for cubic lattice arrangement have confirmed our belief on the performance of the coupling between the OS series and integral equation approximation. We shall proceed with the random distribution of spheres in the next section.

\section{B. Randomly distributed spheres}

The approximation based on triplet structure factors and Neumann series is now applied to determine the effective conductivity for a random distribution of spheres in equilibrium.

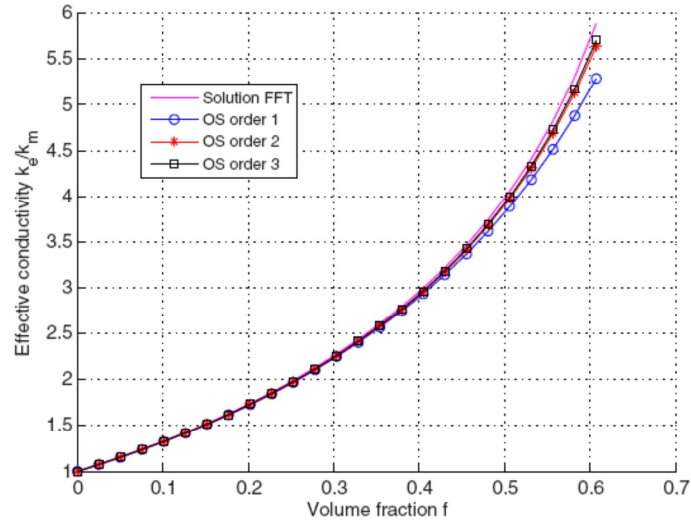

FIG. 3. Comparison the effective conductivity $\left(k_{e} / k_{m}\right)$ of $\mathrm{FCC}$ lattice structure between the approximation OS and FFT complete solution with ratio $k_{i} / k_{m}=100$.

These results are then compared with previous works on the same system from the literature. The first one is the Torquato expression [11] (TO) based on another expansion [10] that gives rise to the microstructure parameter $\zeta$. The latter is intrinsically linked to three-point correlation function and can be evaluated from KSA approximation Eq. (41) or by a direct simulation [30]. As mentioned earlier, this series expansion can yield good result by keeping two or three leading terms but does not guarantee the convergence of the whole series, especially near the percolation limit. Regarding numerical simulations, Bonnecaze and Brady [13,14] (BB) using multipole expansions of particle potential and Kim and Torquato [12] (KT) using random-walk method have obtained precise estimations of the effective conductivity of the system.

It is clear that our OS-1 approximation coincides with the Clausius-Mossoti expression. This is a good starting point since one can expect that higher-order approximations will improve the latter. From results given in Table II, we find that

TABLE II. The effective conductivity $\left(k_{e} / k_{m}\right)$ of randomly distributed spheres. Comparison of the second-order OS-2 estimations (with and without $S^{(3)}$ approximation) and Torquato's expression [11] (TO). Kim and Torquato [12] (KT), the simulation data of Bonnecaze and Brady [13] (BB). The Clausius-Mossoti (CM) approximation coincides with first-order OS approximation OS-1 $(n=1)$.

\begin{tabular}{cccccccc}
\hline \hline \multicolumn{1}{c}{$k_{i} / k_{m}$} & $f$ & $\mathrm{CM}$ & $\begin{array}{c}\text { OS-2 } \\
\text { (approx.) }\end{array}$ & $\begin{array}{c}\text { OS-2 } \\
\text { (exact) }\end{array}$ & TO [11] & KT [12] & BB [13] \\
\hline \multirow{6}{*}{$\infty$} & 0.10 & 1.333 & 1.343 & 1.344 & 1.35 & 1.34 & 1.35 \\
& 0.20 & 1.750 & 1.774 & 1.793 & 1.82 & 1.83 & 1.82 \\
& 0.30 & 2.286 & 2.336 & 2.438 & 2.46 & 2.48 & 2.53 \\
& 0.40 & 3.000 & 3.101 & 3.327 & 3.36 & 3.42 & 3.59 \\
& 0.50 & 4.000 & 4.210 & 4.560 & 4.69 & 4.78 & 4.97 \\
& 0.10 & 1.243 & 1.248 & 1.248 & 1.25 & 1.25 & 1.25 \\
10 & 0.20 & 1.529 & 1.540 & 1.545 & 1.55 & 1.54 & 1.54 \\
& 0.30 & 1.871 & 1.892 & 1.924 & 1.93 & 1.93 & 1.89 \\
& 0.40 & 2.286 & 2.323 & 2.389 & 2.39 & 2.41 & 2.30 \\
& 0.50 & 2.800 & 2.866 & 2.951 & 2.97 & 3.02 & 2.82 \\
\hline \hline
\end{tabular}


our OS-2 estimation with or without $S^{(3)}$ approximation is in very good agreement with the numerical results issued from previous works at moderate contrast $k_{i} / k_{m}<10$ for whole range of volume fraction $f$. For infinite contrast $k_{i} / k_{m}=\infty$, a significant deviation can be observed at $f=0.5$ for OS- 2 with $S^{(3)}$ approximation. This can be explained from the fact that, near the jamming state $f=0.5$, the difference between the theoretical Percus-Yevick (PY) solution and the RDF curves have been found already significant $[13,14]$, while the OS-2 estimation with $S^{(3)}$ approximation relies heavily on the PY solution. In this case, the direct computation based on the real samples should be used. Indeed, numerical results have shown that the direct calculation is in very good agreement with the literature results even for the case of infinite contrast and volume fraction as large as $f=0.5$.

\section{FINAL REMARKS AND DISCUSSION}

To summarize, we have developed an estimation scheme for conductivity of composite materials made of spherical particles and matrix. To do that, we derive first the optimal integral equation on polarization $p$ from a family of integra equations of the same type, i.e., the one whose corresponding Neumann series converges the fastest. Then, a new integral equation is constructed including the first $n-1$ terms of the series and an integral operator of power $n$ acting on the polarization. By applying approximations to the latter, one can expect a good estimation of the average polarization and hence the effective conductivity. Indeed, numerical examples have provided a supporting evidence for this conclusion.

Another interesting result of the present work concerning random media is that we have established the statistical connection between all terms of the Neumann series, solution of the optimized integral equation, with the structure factors, the triplet structure factors and higher-order structure factors, etc. These quantities are very important since they provide useful information on the local distribution of particles. For the case of colloidal suspensions, they can be obtained experimentally via the scattering techniques [31] or via particle simulation methods [32]. Such a dual formulation, using wave-vector formulation instead of a formulation directly in the physical space, has thus given an alternative way to the use of correlation function [6] in order to characterize the properties of random media.

Based on the estimation of the remainder of the Neumann series that is closely related to FFT numerical method, the present method improves the limitations of the effective medium approximation. It is known that the latter fails when the inclusion strongly interact especially at high inclusion volume fraction and high contrast ratio. Without limiting to spherical inclusions, the present method is general enough to deal with complex microstructures, for example, those constituted of cylindrical, ellipsoidal shape inclusions, which will be subject to our future study.

\section{ACKNOWLEDGMENTS}

We thank Mr. Van-Huyen $\mathrm{Vu}$ for his help constructing the random hard-sphere samples. One of authors, Q.-D.T., would like to thank the program Hoa Sen Lotus (Project 30569SK) and the organization Campus France for the travel support.

\section{APPENDIX A: OPERATOR NORM MINIMIZATION}

The norm of the self-adjoint operator $\boldsymbol{B}$ is the maximal eigenvalue $\lambda$ satisfying

$$
\boldsymbol{B} v=\lambda v .
$$

Making use of Eq. (16), we have the following relations in Fourier space:

$$
\left[\lambda_{1} \alpha-\lambda\right] \boldsymbol{G}(\xi) \boldsymbol{v}(\xi)+\left[\lambda_{2} \beta-\lambda\right] \boldsymbol{H}(\xi) \boldsymbol{v}(\xi)=0 .
$$

Since $\boldsymbol{G}(\xi)$ and $\boldsymbol{H}(\xi)$ are projection operators, there are two possibilities:

$$
\boldsymbol{G}(\xi) v(\xi)=0, \quad \lambda=\lambda_{2} \beta \quad \text { or } \quad \boldsymbol{H}(\xi) v(\xi)=0, \quad \lambda=\lambda_{1} \alpha .
$$

As a result, the operator norm $\|\boldsymbol{B}\|$ admits the expression

$$
\|\boldsymbol{B}\|=\max \left\{\left|\lambda_{1} \alpha\right|,\left|\lambda_{2} \beta\right|\right\} \text {. }
$$

Optimizing $\|\boldsymbol{B}\|$ with $\lambda_{1}$ and $\lambda_{2}$ and accounting for $\lambda_{1}+\lambda_{2}=$ 1 , we have

$$
\lambda_{1}=\frac{\beta}{\beta-\alpha}, \quad \lambda_{2}=\frac{-\alpha}{\beta-\alpha}, \quad\|\boldsymbol{B}\|=\frac{|\alpha \beta|}{|\beta-\alpha|} .
$$

Regarding the operator $\boldsymbol{B} \chi$ in the Neumann series, we can estimate its norm via the inequality from the previous result,

$$
\|B \chi v\| \leqslant\|B\|\|\chi v\| \leqslant\|B\|\|\chi\|\|v\| \leqslant\|B\|\|v\| .
$$

From this sequence of inequalities, we conclude that

$$
\|\boldsymbol{B} \chi\| \leqslant\|\boldsymbol{B}\|=\frac{|\alpha \beta|}{|\beta-\alpha|}=\left|\frac{k_{i}-k_{m}}{k_{i}+k_{m}}\right|<1,
$$

which ensures the convergence of the series.

\section{APPENDIX B: TRIPLET STRUCTURE FACTOR APPROXIMATION}

Using the definition of the triplet structure factor and after some mathematical manipulations, we obtain the expression for $S^{(3)}\left(-\xi, \xi^{\prime}\right)$,

$$
\begin{aligned}
S^{(3)}\left(-\xi, \xi^{\prime}\right) & =\frac{1}{N}\left\langle\sum_{i} e^{i \xi \cdot x_{i}} \sum_{j} e^{-i \xi^{\prime} \cdot x_{j}} \sum_{k} e^{-i\left(\xi-\xi^{\prime}\right) \cdot x_{k}}\right\rangle \\
& =1+\frac{1}{N}\left\langle\sum_{i \neq k} e^{i \xi \cdot\left(x_{i}-x_{k}\right)}\right\rangle+\frac{1}{N}\left\langle\sum_{j \neq k} e^{-i \xi^{\prime} \cdot\left(x_{j}-x_{k}\right)}\right\rangle+\frac{1}{N}\left\langle\sum_{i \neq j \neq k} e^{i \xi \cdot\left(x_{i}-x_{k}\right)} e^{-i \xi^{\prime} \cdot\left(x_{j}-x_{k}\right)}\right\rangle,
\end{aligned}
$$


or equivalently,

$$
S^{(3)}\left(-\xi, \xi^{\prime}\right)=S(\xi)+S\left(\xi^{\prime}\right)-1+\frac{1}{N}\left\langle\sum_{i \neq j \neq k} e^{i \xi \cdot\left(x_{i}-x_{k}\right)} e^{-i \xi^{\prime} \cdot\left(x_{j}-x_{k}\right)}\right\rangle .
$$

Here, $\boldsymbol{x}_{i}, \boldsymbol{x}_{j}$, and $\boldsymbol{x}_{k}$ are, respectively, the locations of the particles $i, j$, and $k$. We shall now continue with the last term in Eq. (B2) that is equal to

$$
\begin{aligned}
& \frac{1}{N} \iiint e^{i \xi\left(x-x^{\prime \prime}\right)} e^{-i \xi^{\prime}\left(x^{\prime}-x^{\prime \prime}\right)}\left(\sum_{i \neq j \neq k} \delta\left(x-x_{i}\right) \delta\left(x^{\prime}-x_{j}\right) \delta\left(x^{\prime \prime}-x_{k}\right)\right) d x d x^{\prime} d x^{\prime \prime} \\
& =\frac{\bar{\rho}^{3}}{N} \iiint e^{i \xi x} e^{-i \xi^{\prime} x^{\prime}} g^{(3)}\left(x+x^{\prime \prime}, x^{\prime}+x^{\prime \prime}, x^{\prime \prime}\right) d x d x^{\prime} d x^{\prime \prime} \\
& \quad=\bar{\rho}^{2} \iint e^{i \xi x} e^{-i \xi^{\prime} x^{\prime}} g^{(3)}\left(x, x^{\prime}\right) d x d x^{\prime} \simeq \bar{\rho}^{2} \iint e^{i \xi x} e^{-i \xi^{\prime} x^{\prime}} g\left(x-x^{\prime}\right) g(x) g\left(x^{\prime}\right) d x d x^{\prime},
\end{aligned}
$$

with $\bar{\rho}=\langle\rho(\boldsymbol{x})\rangle=N / V$ being the average particle density. To derive the last equation of Eq. (B3), we have applied the KSA scheme to $g^{(3)}$. This is the exact expression of $S^{(3)}\left(-\xi, \xi^{\prime}\right)$ based on KSA. However, this expression is still difficult to use since it is a double integral in 3D space. For simplification, we approximately replace $g\left(x-x^{\prime}\right)$ with its average 1 and obtain

$$
\bar{\rho}^{2} \iint e^{i \xi x} e^{-i \xi^{\prime} x^{\prime}} g\left(\boldsymbol{x}-\boldsymbol{x}^{\prime}\right) g(\boldsymbol{x}) g\left(\boldsymbol{x}^{\prime}\right) d \boldsymbol{x} d \boldsymbol{x}^{\prime} \simeq \bar{\rho}^{2} \iint e^{i \boldsymbol{\xi} x} e^{-i \boldsymbol{\xi}^{\prime} x^{\prime}} g(\boldsymbol{x}) g\left(\boldsymbol{x}^{\prime}\right) d \boldsymbol{x} d \boldsymbol{x}^{\prime}=[S(\boldsymbol{\xi})-1]\left[S\left(\boldsymbol{\xi}^{\prime}\right)-1\right] .
$$

Finally, the triplet structure factor $S^{(3)}\left(-\xi, \xi^{\prime}\right)$ can be recast in the approximate simplified form of Eq. (43).

[1] J. Maxwell, A Treatise on Electricity and Magnetism (Clarendon Press, Oxford, 1892), Vol. 1

[2] L. Landau and E. Lifshitz, Electrodynamics of Continuous Media (Pergamon Press, Oxford, 1984).

[3] E. Sanchez-Palencia, Nonhomogeneous Media and Vibration Theory, Lecture Notes in Physics, Vol. 127 (Springer Verlag, Berlin, 1980).

[4] G. W. Milton, The Theory of Composites (Cambridge University Press, Cambridge, 2002), Vol. 6.

[5] S. Nemat-Nasser and M. Hori, Micromechanics: Overall Properties of Heterogeneous Materials (Elsevier, Amsterdam, 1999).

[6] S. Torquato, Random Heterogeneous Materials: Microstructure and Macroscopic Properties (Springer Science \& Business Media, Berlin, 2002), Vol. 16.

[7] Z. Hashin and S. Shtrikman, J. Appl. Phys. 33, 3125 (1962).

[8] D. J. Bergman, Phys. Rev. B 19, 2359 (1979).

[9] N. Phan-Thien and G. W. Milton, Proc. R. Soc. London A 380, 333 (1982).

[10] W. Brown, J. Chem. Phys. 23, 1514 (1955).

[11] S. Torquato, J. Appl. Phys. 58, 3790 (1985).

[12] I. Kim and S. Torquato, J. Appl. Phys. 69, 2280 (1991).

[13] R. Bonnecaze and J. Brady, Proc. R. Soc. London A 432, 445 (1991).

[14] R. Bonnecaze and J. Brady, Proc. R. Soc. London A 430, 285 (1990).

[15] Q. D. To, G. Bonnet, and V. T. To, Proc. R. Soc. London A 469. 20120339 (2013)

[16] Q. D. To and G. Bonnet, Phys. Rev. E 91, 023206 (2015).
[17] D. J. Eyre and G. W. Milton, Eur. Phys. J. Appl. Phys. 6, 41 (1999)

[18] Q. D. To and G. Bonnet, Asia Pac. J. Comput. Eng. 1, 5 (2014),

[19] The temperature gradient is defined as $\boldsymbol{e}(\boldsymbol{x})=-\nabla T$, where $T$ is the temperature. Introducing temperature is not necessary since it does not affect the solution of our problem .

[20] S. Nemat-Nasser. T. Iwakuma, and M. Hejazi, Mech. Mater. 1. 239 (1982).

[21] L. Ornstein and F. Zernike, Proc. Acad. Sci. Amsterdam 17, 793 (1914).

[22] J. K. Percus and G. J. Yevick, Phys. Rev. 110, 1 (1958).

[23] M. Wertheim. Phys. Rev. Lett. 10, 321 (1963).

[24] J. G. Kirkwood, J. Chem. Phys. 3, 300 (1935).

[25] J.-P. Hansen and I. R. McDonald, Theory of Simple Liquids (Academic Press, San Diego, 2006).

[26] J.-L. Barrat, J.-P. Hansen, and G. Pastore, Phys. Rev. Lett. 58 2075 (1987).

[27] A. R. Denton and N. W. Ashcroft, Phys. Rev. A 39, 426 (1989).

[28] D. Rapaport, The Art of Molecular Dynamics Simulation (Cambridge University Press, Cambridge, 2004).

[29] V. Monchiet and G. Bonnet, Int. J. Numer. Meth. Eng. 89, 1419 (2012).

[30] C. Miller and S. Torquato, J. Appl. Phys. 68, 5486 (1990).

[31] R. Borsali and R. Pecora, Soft-Matter Characterization (Springer Science \& Business Media, Berlin, 2008).

[32] M. Allen and D. Tildesley, Computer Simulation of Liquids (Oxford University Press, Oxford, 1989). 